\title{
Effects of Tween 80 on Degradation of Benzo[â]pyrene in Soils Growing Sudan Grass
}

\author{
Sheng-wang PAN ${ }^{1, a}$, Zhi-hua LEl ${ }^{2, b}$, Yun-xiao $\mathrm{WU}^{3, c^{*}}$ and Mao-ping \\ $\mathrm{HE}^{1, \mathrm{~d}}$
}

${ }^{1}$ School of Urban and Rural Construction, Chengdu University, Chengdu-610106, China

\author{
${ }^{2}$ School of Medical Technics, Xinyang Vocational \& Technical College, \\ Xinyang-465350, China \\ ${ }^{3}$ Colege of Life Science, Yan'an University, Sanxi-716000, China \\ apanwang@swu.edu.cn, bzhhlei@163.com, cyxwu@163.com, ${ }^{\mathrm{d}} 736342142 @ q q . c o m$ \\ ${ }^{*}$ Corresponding Author
}

Keywords: Phytoremediation, Surfactants, SEPR, Benzo[A]Pyrene, Tween 80.

\begin{abstract}
Phytoremediation is becoming a cost-effective technology for the in-situ clean up of sites polluted with hydrophobic organic contaminants (HOCs). The major factors limiting phytoremediation are the mass transfer, rate of plant uptake, and microbial biodegradation of HOCs. To evaluate the potential of surfactants to enhance phytoremediation for HOC-contaminated sites, the efficacy of Sudan grass, at the absence or presence of polyoxyethylene sorbitan monooleate (Tween 80), on the degradation of benzo[â]pyrene $(\mathrm{BaP})$ in soils were investigated, and mechanisms of surfactant-enhanced phytoremediation (SEPR) were discussed. Results showed that the presence of Tween 80 enhanced dissipation of $\mathrm{BaP}$ at initial contents ranging from 20.24 to $321.42 \mathrm{mg} / \mathrm{kg}$. During the 70-d SEPR-experiments, about 731.84 469.99\%o of $\mathrm{BaP}$ was removed from planted soils, only $242.28 \sim 122.79 \%$ degradation of $\mathrm{BaP}$ occurred in unplanted ones. With the presence of Tween 80 , the dissipation ratios of $\mathrm{BaP}$ in planted ones were increased up to $863.94 \sim 609.63 \%$, which was $139.64 \sim$ $190.16 \%$ higher than those in corresponding soils without surfactant. Among all possible pathways, contribution of plant-microbial interactions on dissipation of $\mathrm{BaP}$ was the most significant, either at the presence $(456.73 \%$ ) or absence $(515.58 \%$ ) of Tween 80 , were the primary means of contaminant degradation. Results suggested SEPR may be a feasible way for reinforcing removal of HOCs in contaminated sites.
\end{abstract}

\section{Introduction}

Phytoremediation is a promising approach to soil remediation due to its convenience, cost-effectiveness and environmental acceptability[1]. Plants may contribute to the dissipation of organic contaminants through an increase in the number of microbes, improvement of soil properties and structure, promotion of humification and adsorption of pollutants in the rhizosphere, but the impact of each process has not been clearly elucidated, and remediation efficacy varies greatly among plant species depending on the differences of soil conditions and physicochemical nature of contaminants [2]. However, there also exist many limitations for large-scale application of this technology. One serious limitation is that HOCs in soils usually exhibit low bioavailability to both micro- organisms and plants due to their strong affinity to the soil matrix, especially to soil organic matter, which would limit the application of phytoremediation $[3,4]$. 
Surfactants are amphiphilic molecules with a hydrophobic portion (usually a long hydrocarbon chain) and a hydrophilic head group. Surfactant is an abbreviation for 'surface active agent', so named because these molecules tend to migrate to surfaces and interfaces or create new molecular surfaces by forming aggregates. The critical micelle concentration (CMC), the surface and interfacial tension (IFT), and the hydrophile-lipophile balance (HLB) are the three main parameters that help characterize surfactant activity in solutions. There are four general classes of surfactants, which are classified by the charge on the polar head group: anionic, nonionic, cationic, zwitterionic [5]. Surfactants may aid in remediation by decreasing the surface tension of the water and efficiently solubilizing or otherwise mobilizing nonaqueous phase liquids. As the surfactant concentration increases, the surface tension of the solution decreases until the CMC is reached. The addition of surfactants to a soil contaminated with HOCs might increase the mass transfer and the bioavailability of these compounds, facilitating their degradation. The ability of the surfactants to increase desorption, apparent aqueous solubility, and microbial bioavailability of HOCs has been well documented. Recently, it has been shown that addition of surfactants facilitates the uptake of HOCs by plants. These results indicate a promising opportunity to use surfactants to enhance phytoremediation efficacy.

In this study, the effect of Sudan grass (Sorghum sudanense), at the absence or presence of surfactants, on the dissipation of benzo[â]pyrene $(\mathrm{BaP})$ in the spiked soils was investigated and each removal pathway of $\mathrm{BaP}$ in the process of remediation was compared in order to understand the potential of surfactant-enhanced phytoremediation (SEPR) of HOC-contaminated soils. Furthermore, the mechanisms of SEPR for soil HOCs were assessed by considering experimental observations.

\section{Materials and Methods}

\section{Experimental Materials}

Benzo[â]pyrene $(\mathrm{BaP})$, as representative of HOCs, was purchased from Sigma (St. Louis, MO), and were HPLC grade with a purity $>98.5 \%$, molecular weights of $252.31 \mathrm{~g} / \mathrm{mol}$ and 6.0 of $\log K_{o w}\left(K_{o w}\right.$, octanol-water partition coefficient). Polyoxyethylene sorbitan monooleate(Tween 80), as representative of surfactants, was purchased from Kasei Kogyo Co. Ltd with a purity $\geq 98 \%$, molecular weights of 1310 $\mathrm{g} / \mathrm{mol}, 15$ of HLB and CMC of $15.72 \mathrm{mg} / \mathrm{L}$. Four percent (vol/vol) was selected in this study because it showed a good solubilization for hydrophobic organic compounds and gave the best removal efficiency in a previous study.

Soils tested were collected from $6 \sim 18 \mathrm{~cm}$ horizontal with $\mathrm{pH} 6.78,1.46 \%$ organic matters and originally free of PAHs. After being air-dried and sieved through a $2 \mathrm{~mm}$ mesh, soils were spiked with $\mathrm{BaP}$ in acetone. When acetone evaporated off, spiked soils were progressively mixed with unpolluted soils and homogenized. The initial $\mathrm{BaP}$ in soils were measured and followed as: $\mathrm{C}_{0}$ (free PAHs), $\mathrm{C}_{1}(20.2 \pm 0.94 \mathrm{mg} / \mathrm{kg}$ ), $\mathrm{C}_{2}(39.58 \pm 1.51 \mathrm{mg} / \mathrm{kg}), \mathrm{C}_{3}(79.86 \pm 2.37 \mathrm{mg} / \mathrm{kg}), \mathrm{C}_{4}(160.64 \pm 3.05 \mathrm{mg} / \mathrm{kg})$ and $\mathrm{C}_{5}$ $(321.42 \pm 4.93 \mathrm{mg} / \mathrm{kg})$. Treated soils were packed into pots $(2.0 \mathrm{~kg})$ and equilibrated $4 \mathrm{~d}$ under $45 \%$ of the water holding capacity (WHC).

After surface sterilized in $10 \% \mathrm{H}_{2} \mathrm{O}_{2}$ for $10 \mathrm{~min}$ and rinsed with distilled water, Sudan grass seeds were germinated and grown in moist perlite, and seedlings of uniform size were transplanted to the designated greenhouse pots $7 \mathrm{~d}$ after emergence.

\section{Experimental Design}

To assess the effects of addition of surfactants (Tween 80) to enhance 
phytoremediation for $\mathrm{BaP}$ in soils, we used two separate greenhouse experiments in Chengdu University in 2016. In the first experiment, four different treatments with five replicates were performed: i ) treatments $1\left(\mathrm{CK}_{1}\right)$, unplanted microbe-inhibited soils, where $0.1 \% \mathrm{NaN}_{3}$ was added to inhibit the microbial activity[6], ii ) treatments $2\left(\mathrm{CK}_{2}\right)$, unplanted soils, iii) treatments $3\left(\mathrm{TR}_{3}\right)$, planted microbe-inhibited soils added $0.1 \% \mathrm{NaN}_{3}$, and iv) treatments 4 ( $\left.\mathrm{TR}_{4}\right)$, planted soils. Seedlings were thinned 5 $\mathrm{d}$ after transplanting to 8 plants per pot. WHC was checked and adjusted regularly with sterilized water to $50 \%[6]$

In the second experiment, all treatments and culture conditions were the same as the former besides addition of Tween 80 . Based on soil properties and weights per pot, $300 \mathrm{ml}$ of $4 \%$ ( $\mathrm{vol} / \mathrm{vol})$ Tween 80 were added to soils at the beginning of experiments, and then equilibrated $4 \mathrm{~d}$ under $45 \%$ WHC prior to transplanting.

Soils and plants were destructively sampled after the $70 \mathrm{~d}$ SEPR-experiments. Shoot and root tissues, separated from soil, were washed with distilled water and dried with filter paper. The plant materials and soil samples were stored at $-40.0{ }^{\circ} \mathrm{C}$ to prevent microbial degradation of contaminants. An aliquot of each soil or plant sample was weighed, dried at $105.0{ }^{\circ} \mathrm{C}$ for $24 \mathrm{~h}$, and weighed again.

\section{Determination of HOCs}

The HOCs contents were determined using the high performance liquid chromatography method with ultraviolet detection after a preliminary sample treatment with ultrasonic techniques, and the detailed methods to extract $\mathrm{BaP}$ have been described[7].

Prior to use, all methods were tested for efficiency of recovery. For PAHs-amended soils, plant tissue samples, recovery averaged $93.52 \%(\mathrm{n}=9, \mathrm{RSD}<6.37 \%$; RSD, relative standard deviation) and $93.23 \%(\mathrm{n}=9, \mathrm{RSD}<5.49 \%)$, respectively.

\section{Data Analyses}

The obtained data were analyzed using SPSS version 13.0, and levels of significance were assessed with Duncan's multiple-range test $(P<0.05)$.

For every treated pot, the dissipation rate $(D)$ of $\mathrm{BaP}$ in soils was calculated as $D=$ $\left(C_{0}-C_{\mathrm{t}}\right) \times 1000 \% / C_{0}$, where $C_{0}$ was the initial contents of BaP in soils, and $C_{\mathrm{t}}$ denoted residual $\mathrm{BaP}$. For given biotic \& abiotic factor $i$, its contribution rate $\left(T_{i}\right)$ to the dissipation of PAHs in the process of phytoremediation was expressed as $T_{\mathrm{i}}=D_{i}$ $\times 1000 \% / W \cdot C_{0}$, where $D_{i}$ was the removed amount of PAHs by given factor $i$, and $W$ denoted the weight of spiked soils in pot. Obviously, the $D$ value should be theoretically equal to the sum of contribution rates of all factors [6, 7].

\section{Results}

\section{Plant Biomass}

As shown in Fig.1, Sudan grass showed no signs of stress and produced abundant biomass in spiked soils. Throughout the experiment, the total mass of Sudan grass in soils with low $\left(\mathrm{C}_{1}\right)$, medium $\left(\mathrm{C}_{3}\right)$ and high $\left(\mathrm{C}_{5}\right)$ initial contents of $\mathrm{BaP}$ were almost equal to those of unspiked soils $(1.43 \mathrm{~g})$ with the almost same ratios of root to shoot biomass $(0.284)$. Though biomass of plants grown in high pollution level soils $\left(\mathrm{C}_{5}\right)$ was slightly decreased, all plants tested did not also show any visible sign of toxicity, indicating that establishment of vegetation in these soils is feasible. The weights of roots or shoots of Sudan grass growing in soils at the presence of 4\% Tween 80 (PT) were on average $16.6 \%$ greater than those grown in spiked soils, but the 
differentiation in the total biomass of seedlings growing in variously spiked soils.
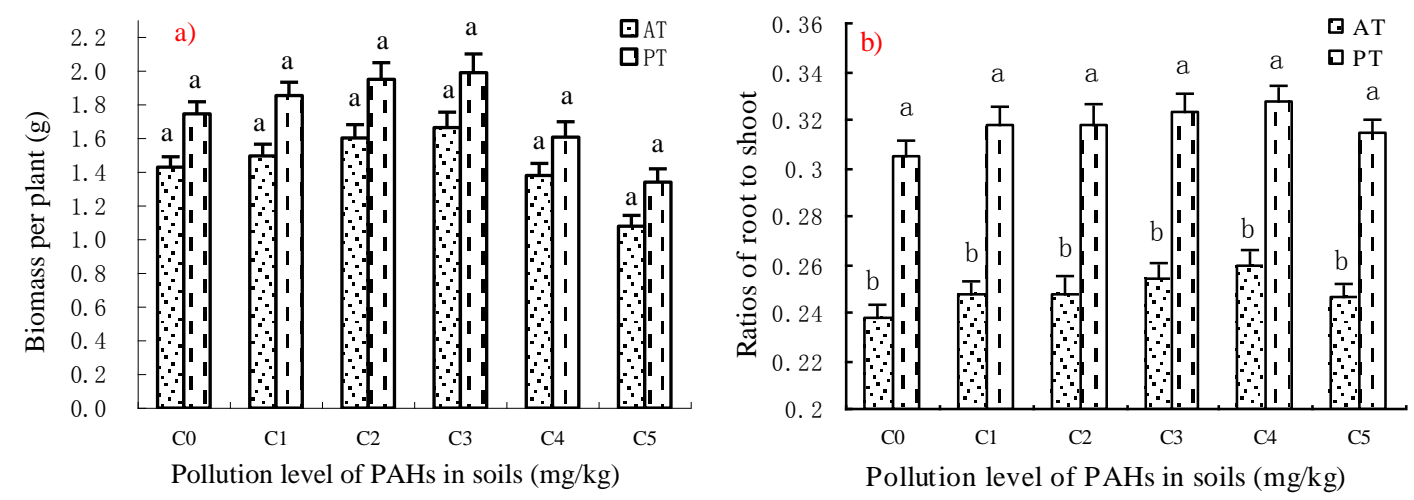

Fig.1 Biomass and ratios of root to shoot of plants grown in soils (PT and AT denoted the BaP-spiked soils at the presence and absence of $4 \%$ Tween 80 , respectively)

$\left(\mathrm{C}_{0} \sim \mathrm{C}_{5}\right)$ between at the presence of Tween $80(\mathrm{PT})$ and absence of that (AT) was insignificant while their pollution levels in soils were the same, individually. However, their ratios of root to shoot biomass tended to be significantly greater at the presence of Tween $80(n=30, P<0.05)$, indicating that the presence of surfactants (Tween 80$)$ was more favorable to the growth of root systems.

\section{Dissipation of HOCs}

On the whole, residual $\mathrm{BaP}$ in soils at the presence of $4 \%$ Tween 80 (PT) were always lower than those at the absence (AT) for the same treatment. Within the same group, the removal of $\mathrm{BaP}$ in treated soils showed a consistent descending order: $\mathrm{CK}_{1} \leqslant \mathrm{TR}_{3}<\mathrm{CK}_{2}<\mathrm{TR}_{4}$. With an increase of initial contents of $\mathrm{BaP}$ in soils, their $D$ value linearly declined for the same treatment. As shown in Fig.2, the presence of plants apparently enhanced the removal of $\mathrm{BaP}$ in soils at the presence or absence of Tween 80. During the experiment, approximately $757.33 \%$ (in a range of 863.94 $609.63 \%$ ) of $\mathrm{BaP}$ was removed from the $\mathrm{TR}_{4}$ with surfactants as compared with 595.19\%o (731.84 469.99\%o) degradation without surfactants, while only $182.38 \%$ o (242.28 122.79\%o) removal occurred in $\mathrm{CK}_{2}$ without surfactants at concentrations of $\mathrm{C}_{1} \sim \mathrm{C}_{5}$. Of the spiked soils, the BaP dissipation rates in $\mathrm{TR}_{4}$ with Tween 80 were the highest, indicating that addition of surfactants to phyto- remediation system could enhance removal of $\mathrm{BaP}$ to a certain extent.

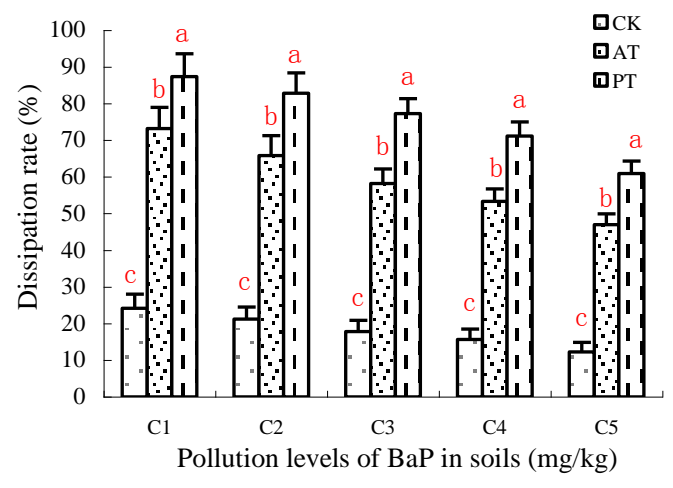

Fig.2 Dissipation rates of BaP in soils under different treatments (CK, PT and AT represent unplanted soils, and planted soils at the presence and absence of $4 \%$ Tween 80 , respectively)

Though the dissipation of BaP in treated soils with surfactants were larger than those without surfactants, the extent of enhanced removal varies greatly among five pollution levels. As shown in Fig.3, the enhanced dissipation of $\mathrm{BaP}$ in soils with 
medium pollution level $\left(\mathrm{C}_{3}\right)$ was the highest, which were up to $140.16 \%$ in the vegetated soils $\left(\mathrm{TR}_{4}\right)$. Comparatively, fewer extents of removal enhanced occurred in soils with low $\left(C_{1}\right)$ or high $\left(C_{5}\right)$ pollution level, only $92.14 \%$ and $89.64 \%$ additional removal, respectively. On a whole, extents of enhanced dissipation of BaP were up to $114.15 \%$ o during the $70 \mathrm{~d}$ SEPR-experiments.

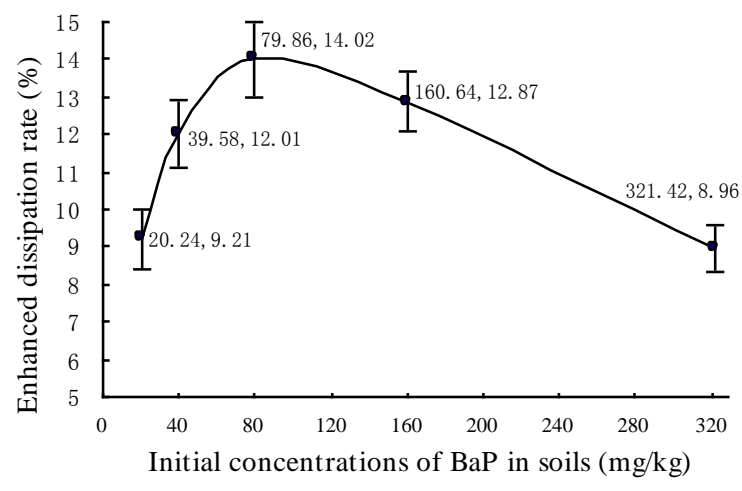

Fig.3 Enhanced dissipation rates of $\mathrm{BaP}$ in soils at different pollution levels by Tween 80

\section{HOCs Accumulated in Plant Tissues}

As expected, accumulation levels of $\mathrm{BaP}$ in plant tissues have a positive correlation with their pollution levels; $\mathrm{BaP}$ contents in plant tissues are always lower in soils at the presence of $4 \%$ Tween 80 (PT) than those at the absence (AT). With the increase of pollution levels in soil, $\mathrm{BaP}$ contents in roots and shoots of plants growing in soils without surfactants increased from 14.17 to $72.26 \mathrm{mg} / \mathrm{kg}$ and from 2.08 to 13.79 $\mathrm{mg} / \mathrm{kg}$ while increased from 9.69 to $58.59 \mathrm{mg} / \mathrm{kg}$ and from 1.32 to $10.36 \mathrm{mg} / \mathrm{kg}$ when adding surfactants, respectively.

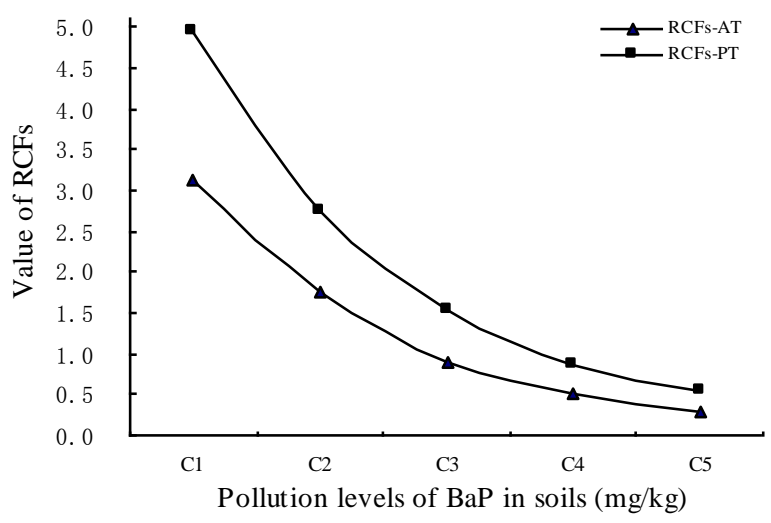

Fig.4 RCFs of BaP as a function of PAHs concentrations in soils (RCFs-PT and RCFs-AT denoted root concentration factors in soils at the presence and absence of Tween 80 , respectively)

Based on residual HOCs in soils and their accumulation levels in roots of plant tissues by the end of the entire SEPR-experiments, RCFs (root concentration factors, defined as the ratio of HOCs concentration in roots and one in soils) of BaP in soils were calculated as shown in Fig.4. With the increase of contaminants concentration in soils, RCFs of BaP tended to decrease. Values of RCFs (0.30 3.14) in plant-soil system without surfactants (AT) were always lower than those $(0.53 \sim 4.95)$ at the presence of surfactants (PT).

\section{Removal Pathways of HOCs}

Removal of HOCs in the plant-soil system attributed to both biotic pathways, e.g., plant accumulation, plant metabolism, microbial degradation and plant-microbial interactions, and abiotic pathways, e.g., leaching, volatilization, photodegradation and 
irreversible sorption, etc. If the variation of abiotic loss of HOCs between planted and unplanted soils was negligible, HOCs removed in $\mathrm{CK}_{2}$ should be equal to the sum of abiotic loss and microbial degradation while loss of HOCs in $\mathrm{CK}_{1}$ attributed to abiotic loss. Thus, removal of BaP in soils could approximately be expressed as

$$
\begin{aligned}
& D_{l}=T_{a} . \\
& D_{2}=T_{a}+T_{m} . \\
& D_{3}=T_{a}+T_{c}+T_{d} . \\
& D_{4}=T_{a}+T_{c}+T_{d}+T_{m}+T_{p m} .
\end{aligned}
$$

Where $D_{1}, D_{2}, D_{3}$ and $D_{4}$ were the removed amount of $\mathrm{BaP}$ in $\mathrm{CK}_{1}, \mathrm{CK}_{2}, \mathrm{TR}_{3}$ and $\mathrm{TR}_{4}$, and $T_{a}, T_{m}, T_{c}, T_{d}$ and $T_{p m}$ represented the contribution of abiotic loss, microbial degradation, plant accumulation, plant metabolism and plant-microbial interactions on removal of $\mathrm{BaP}$, respectively.

As to soils added surfactants, the potential of surfactants in increasing the mass transfer and the bioavailability of these compounds, facilitating their degradation should be also taken into account. Then, enhanced value $\left(\Delta D_{i}^{\mathrm{s}}\right)$ of $\mathrm{BaP}$ in $\mathrm{CK}_{1}, \mathrm{CK}_{2}$, $\mathrm{TR}_{3}$ and $\mathrm{TR}_{4}$, could be respectively expressed as

$$
\begin{aligned}
& \Delta D_{1}{ }^{s}=T_{a}{ }^{s} . \\
& \Delta D_{2}{ }^{s}=T_{a}{ }^{s}+T_{m}{ }^{s} . \\
& \Delta D_{3}{ }^{s}=T_{a}{ }^{s}+T_{c}{ }^{s}+T_{d}{ }^{s} . \\
& \Delta D_{4}{ }^{s}=T_{a}{ }^{s}+T_{c}{ }^{s}+T_{d}{ }^{s}+T_{m}{ }^{s}+T_{p m}{ }^{s} .
\end{aligned}
$$

Where $\Delta D_{i}^{\mathrm{s}}$ represented the enhanced removed value of $\mathrm{BaP}$ in $\mathrm{CK}_{1}, \mathrm{CK}_{2}, \mathrm{TR}_{3}$ or $\mathrm{TR}_{4}$ when adding surfactants, and $T_{a}{ }^{s}, T_{m}{ }^{s}, T_{c}{ }^{s}, T_{d}{ }^{s}$ and $T_{p m}{ }^{\mathrm{s}}$ did the enhanced amount of $T_{a}, T_{m}, T_{c}, T_{d}$ and $T_{p m}$ by surfactants, respectively. Based on $\mathrm{BaP}$ detected in leachate, soils, plant and earthworm tissues, contribution rate of each pathway on removal of BaP was calculated as Table 1.

Table 1. Contributions of each pathway on BaP dissipation at the absence or presence of Tween $80(\%)$

\begin{tabular}{lccccc}
\hline Factors & $\mathrm{C}_{1}$ & $\mathrm{C}_{2}$ & $\mathrm{C}_{3}$ & $\mathrm{C}_{4}$ & $\mathrm{C}_{5}$ \\
\hline Abiotic loss & 32.07 & 29.28 & 25.64 & 22.61 & 18.16 \\
Microbial degradation & $(1.18)$ & $(1.94)$ & $(2.28)$ & $(1.51)$ & $(1.16)$ \\
& 210.23 & 182.62 & 152.25 & 134.34 & 104.62 \\
Plant accumulation & $(15.35)$ & $(27.82)$ & $(37.83)$ & $(34.34)$ & $(18.54)$ \\
& 1.06 & 0.72 & 0.62 & 0.37 & 0.29 \\
Plant metabolism & $(0.00)$ & $(0.00)$ & $(0.00)$ & $(0.00)$ & $(0.00)$ \\
& 49.24 & 39.46 & 21.82 & 9.81 & 5.14 \\
Plant-microbial & $(6.83)$ & $(5.83)$ & $(3.95)$ & $(3.15)$ & $(2.21)$ \\
interactions & 509.24 & 476.51 & 452.19 & 435.83 & 411.78 \\
& $(39.05)$ & $(64.32)$ & $(76.05)$ & $(69.68)$ & $(47.69)$ \\
\hline
\end{tabular}

(Data in bracket were the enhanced $T_{\mathrm{i}}$ of given factor $i$ when adding $4 \%$ Tween 80 )

In the entire SEPR-experiments, no leachate was produced when WHC in soils was maintained at about $45 \%$, and abiotic loss by leaching was insignificant. As seen from Table 1, the $D$ value of $\mathrm{BaP}$ in microbe-inhibited pots, i.e., $\mathrm{CK}_{1}$, was $18.16 \sim 32.07 \%$ o $(\mathrm{M}=25.58 \%$ ), indicating that abiotic loss was a relatively minor pathway for the dissipation of $\mathrm{BaP}$. However, contribution of microbial degradation on removal of contaminants was notable, which was up to $104.62 \sim 210.23 \%$ o $(\mathrm{M}=158.76 \%$ o). In this case, role of plant accumulation in dissipation of $\mathrm{BaP}$ was inappreciable compared to the total loss of HOCs. During the 70-d SEPR-experiments, the amount of BaP accumulated by tall fescue only accounted for $0.29 \sim 1.06 \%$ ( $\mathrm{M}=0.61 \%$ ) from $\mathrm{C}_{1}$ to $\mathrm{C}_{5}$ treatment, which were less than $0.92 \%$ of $\mathrm{BaP}$ degraded averagely while one derived from plant metabolism was in the range of 5.14 49.24\%o $(\mathrm{M}=25.09 \%)$, which were less than $38.22 \%$ of $\mathrm{BaP}$ degraded in soils. Among all removal pathways, the plant- 
microbial interactions contributed the most part for rhizoremediation of HOCs, which removed up to $456.73 \%$ (in a range of 411.78 509.24\%o) from spiked soils averagely, illuminating that the predominant pathway responsible for the dissipation of HOCs in soils was the plant-microbial interactions.

As shown in Table 1, enhanced dissipation of BaP in soils at the presence of $4 \%$ Tween 80 varied greatly among removal factors. On the whole, the enhanced extent of plant-microbial interactions and microbial degradation were higher, which was up to $59.36 \%$ and $26.78 \%$ on average while comparing with the much lower abiotic loss, whose contributions enhanced only accounted for $1.61 \%$. It was notable that the contribution rate of plant accumulation to $\mathrm{BaP}$ was slightly lower in soils at the presence of Tween 80 than in soils without surfactants, which was consistent with the experimental facts that with surfactants, $\mathrm{BaP}$ concentrations in plant tissues were always lowered at the same soil concentrations.

\section{Discussions}

Phytoremediation is a potential 'green', cost-effective, and secure technology that uses plants for the in-situ cleanup of contaminated sites. It is a promising remediation technique for soils contaminated with organic compounds [8]. Plants may assist in the dissipation of HOCs through the release of root exudates. Exudates act as microbial substrates that enhance soil microbial populations and their activity in the vicinity of HOC contamination. Moreover, plant uptake and accumulation may also contribute to the removal of HOCs from the soil environment [9]. In this study, plant-mediated HOCs dissipation was investigated, and loss of $\mathrm{BaP}$ in spiked soils $\left(\mathrm{C}_{1} \sim \mathrm{C}_{5}\right)$ growing Sudan grass was $731.84 \sim 469.99 \%$ of these chemicals, which were 489.54 347.19\%o larger than those in unplanted soils. The high dissipation ratios of HOCs in planted soils, the evident dissipation promotion of these compounds in the presence of vegetation, and the healthy growth of the plant in variously spiked soils suggest the feasibility of remediation of HOCs in soils using Sudan grass.

The addition of surfactants to a soil contaminated with HOCs might increase the mass transfer and the bioavailability of these compounds, facilitating their degradation. The ability of the surfactants to increase desorption, apparent aqueous solubility, and microbial bioavailability of HOCs has been well documented. Recently, it has been shown that addition of surfactants facilitates the uptake of HOCs by plants [10]. In this study, After adding 4\% Tween 80 , the dissipation of $\mathrm{BaP}$ in variously unplanted soils $\left(\mathrm{C}_{1} \sim \mathrm{C}_{5}\right)$ were 731.84 469.99\%o, which were 489.54 347.19\%o higher than those in corresponding soils without surfactants, and in soils with Sudan grass were up to 863.94 609.63\%o, which were 139.64 190.16\% higher than those in corresponding soils without surfactants, respectively. These results indicate a promising opportunity to use surfactants to enhance phytoremediation efficacy.

It is noteworthy that the amount of $\mathrm{BaP}$ accumulated by Sudan grass growing in soils added surfactants were always less than those without surfactants, and $\mathrm{BaP}$ concentrations in plant tissues, irrespective of root and shoot, were always slightly lower at the same soil concentrations, implying that adding surfactants seemed to be beneficial to decreasing HOCs accumulations in plant tissues to a certain extent, which could be especially beneficial for relieving potentially ecological risks.

\section{Conclusions}

In conclusion, adding surfactants apparently reinforced the dissipation of $\mathrm{BaP}$ in planted soils at initial contents ranging from 20.24 to $321.42 \mathrm{mg} / \mathrm{kg}$. During the $70-\mathrm{d}$ 
SEPR-experiments, after adding surfactants, loss of BaP in variously spiked soils $\left(C_{1} \sim C_{5}\right)$ with Sudan grass were up to $863.94 \sim 609.63 \%$, which were 92.06\%o larger than those in corresponding soils without surfactants averagely, which suggested that SEPR may be a feasible way for reinforcing removal of HOCs in contaminated sites. However, it remains to be seen whether the dissipation of HOCs in the plant-soil system adding surfactants is as effective under large-scale field conditions as it appears under laboratory conditions.

\section{Acknowledgements}

This research was financially supported by plan of benefiting the people of science and technology of Chengdu (2015-HM01-00368-SF) and natural science foundation of the education department of Sichuan province (17ZA0091).

\section{References}

[1] H. Yi and D.E. Crowley: Environ. Sci. Technol, Vol.4 (2007), p.4382

[2] S.D. Siciliano, J.J. Germida, K. Banks: Appl. Environ. Microbiol. Vol. 69(2003), p. 483

[3] B.L. Chen, E.J. Johnson, B. Chefetz, L.Z. Zhu, B.S. Xing: Environ. Sci. Technol. Vol. 39 (2005), p.6138

[4] N.S. Eriksen-Hamel, J.K. Whalen: Soil Biol. Biochem. Vol. 40(2008), p.1004

[5] D.H. Lee, D.C. Robert, D.J. Kim, C. Sangil: Environ. Int. Vol. 27(2002), p.681

[6] S.Q. Wei and S.W. Pan: J. Soil Sedimen. Vol.10 (2012), p.886

[7] S.W. Pan, L.J. Yang, S.X. Cao, X. Yuan, M.P. He: Appl. Mechan. Mater. Vol.453 (2014), p.850

[8] M.C. Tejeda-Agredano, S. Gallego, J. Vila, M. Grifoll, J.J. Ortega-Calvo, M. Cantos: Soil Biol. Biochem. Vol. 57 (2013), p.2065

[9] K. Sumia, A. Muhammad, I. Samina, M.K. Qaiser: Chemosphere. Vol. 90 (2013), p.1317

[10] Y.Z. Gao, W.T. Ling, L.Z. Zhu, B.W. Zhao: Pedosphere. Vol. 17 (2013), p.409 